\title{
PRODUÇÃO DE FILMES DE POLIAMIDA 6.6 PELO MÉTODO DE INVERSÃO DE FASES
}

\author{
M. MARQUES ${ }^{1}$, L.A. SOARES ${ }^{1}$, A. P. S. IMMICH ${ }^{1}$ \\ ${ }^{1}$ Departamento das Engenharias, Universidade Federal de Santa Catarina, Campus Blumenau \\ - Blumenau, SC \\ E-mail para contato: m.mariana@grad.ufsc.br
}

\begin{abstract}
RESUMO - As exigências com a segurança em relação aos substratos têxteis é crescente diante das vastas aplicações técnicas possíveis, como por exemplo, em vestuário de proteção, na área automobilística e na aeronáutica. Dentre os possíveis materiais utilizados, os polímeros são altamente requisitados pela sua flexibilidade e resistência. Essas características possibilitam uma construção avançada e diferenciada envolvendo a combinação de tecnologia e ergonomia. Para completar as vantagens desses materiais, faz-se necessário uso de aditivos para melhorar sua propriedades. Neste trabalho são descritos métodos para produzir um filme de poliamida que posteriormente terá sua superfície modificada para aprimorar as prioridades. Para obtenção de uma membrana resistente, com espessura uniforme e superfície íntegra, várias metodologias de formação de filmes foram testadas. Os resultados mostraram que o método de formação de filme e a viscosidade deste são os parâmetros que regulam a integridade e regularidade do filme formado. A adição de um plastificante, como a glicerina, na solução polimérica, também contribui para a resistência e estabilidade do filme. Este filme de poliamida obtido foi a primeira etapa da produção de filmes de poliamidas funcionais, portanto, mais estudos serão realizados com o desenvolvimento de um substrato de alto desempenho funcionalizado para melhorar a resistência à chama do produto para futuras aplicações em vestuário de proteção.
\end{abstract}

\section{INTRODUÇÃO}

A utilização dos materiais têxteis de alto desempenho tem crescido em larga escala nas áreas técnicas. Setores como automobilístico, aeroespacial (Horrocks, 2016 a), medicinal e hospitalar (Rajendran, 2016), construção civil (Rawal, 2016), etc, tem utilizado fibras têxteis substituindo eficientemente matérias-primas tradicionais, como plásticos e metais. O sucesso dessa troca deve-se às características das fibras, geralmente com a alta resistência aliada ao baixo peso, e muitas vezes sendo biodegradável ou biocompatível. A facilidade de conformação e moldagem pela flexibilidade é outro fator que facilita o desenvolvimento de produtos com inovação de design e facilitadores ergonômicos. 
Um dos setores que utiliza de forma massiva essas propriedades dos artigos têxteis é o vestuário de proteção (Kumar, 2014; Holmes e Horrocks 2016 a). Para a manufatura de uma vestimenta que cumpra os requisitos, tanto de segurança quando de ergonomia, materiais cada vez mais avançados são recomendados. Tendo contato direto com o usuário, a roupa de proteção também deve presar pelo conforto e a usabilidade dos uniformes. A introdução de uma fina membrana, com tratamento especial adequado para cada situação, de entre camadas (Crown e Batcheller, 2016), promove reforço e é uma solução de baixo comprometimento para a usabilidade do equipamento.

Dentre os materiais têxteis mais utilizadas para a construção de vestuário de proteção encontramos polímeros sintéticos, como por exemplo, poliésteres e poliamidas. Elas se destacam, quando comparado a materiais naturais, na facilidade de modificação, sendo possível manipular suas características através de aditivos e melhorar propriedades requisitadas em aplicações onde o alto desempenho seja requerido.

A poliamida é um polímero sintético amplamente conhecido e aplicado no meio têxtil principalmente pela sua alta elasticidade, conferindo conforto ao usuário. Além desta característica, Deopura et al. (2008) destaca também as propriedades de resistência a abrasão e a alta resiliência. Entretanto, uma característica que é relevante no caso de aplicação em vestuário de proteção é a resistência ao fogo que a matéria-prima possui, e este é um aspecto a ser melhorado na poliamida. Atualmente existe no mercado opções de poliamidas que são melhoradas para garantir a resistência em contato com o calor, porém, são de alto custo. Neste sentido, observa-se oportunidade de desenvolver materiais de alto desempenho e que possuam um custo viável em relação ao seu benefício, tornando mais acessível o uso de proteção.

O objetivo deste trabalho é produzir filmes de poliamida para posterior modificação e funcionalização, para serem usados como corpos de prova de ensaios de resistência térmica e mecânica, a fim de produzir filmes e fibras de alto desempenho.

\section{METODOLOGIA}

\subsection{Materiais}

O polímero utilizado para construção das membranas foi a poliamida 66 , fornecedor Rodhia. Como solvente para a solução utilizou-se ácido fórmico, com grau de pureza de 88 $\%$, da marca Biotec. O agente plastificante utilizado foi a glicerina, marca Lafan.

\subsection{Construção da membrana}

O polímero foi solubilizado em ácido fórmico, com concentração de $10 \%$, (massa/massa), em agitação magnética constante e temperatura ambiente, até completa dissolução.

A solução polimérica foi uniformemente espalhada em placas suportes de vidro, plástico e metal, formando um filme fino. Para solidificação do filme, as placas foram imersas em água destilada, em temperatura ambiente, para através do método de inversão de fases, descrito por Medeiros et. al. (2013), solidificar o filme. A secagem da membrana ocorreu em temperatura ambiente overnight. 


\section{RESULTADOS E DISCUÇÕES}

Apesar de a fabricação de filmes ser uma técnica amplamente estudada e aplicada, a execução em laboratório, da produção de filmes de poliamida, não foi trivial e demandou vários testes até se obter uma membrana suficientemente homogênea e íntegra.

O método de espalhamento da poliamida na placa formadora de filme foi um fator importante para se obter uma membrana mais resistente e menos quebradiça. Testes feitos com aplicação em pincel não obtiveram bons resultados, pois os filmes formados eram demasiadamente finos, estriados devido às cerdas do pincel, inviabilizando a remoção da membrana intacta. O método de espalhamento com espátula resultou num filme com espessura uniforme, de $0,03 \mathrm{~mm}$, que possibilitou a retirada da membrana sem danos.

Outro fator importante na formação do filme foi a viscosidade da solução polimérica. Filmes muito viscosos (concentração de $10 \%$ ) resultavam em filmes quebradiços e pouco flexíveis, com mostra a Figura 1.

Figura 1 - Filme quebradiço de poliamida 66 devido à alta viscosidade da solução.

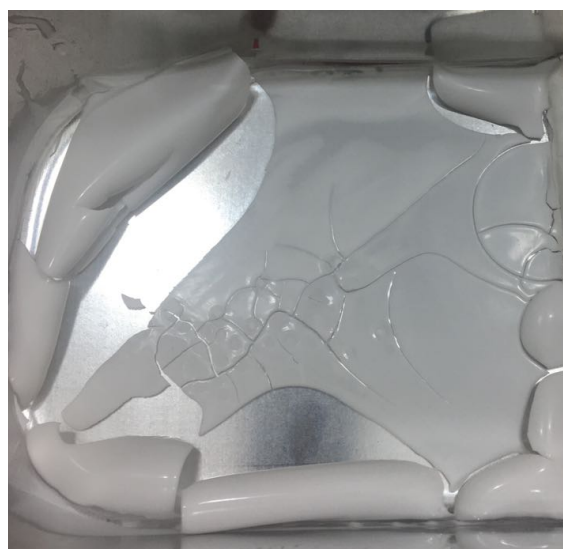

Soluções menos viscosas, com concentração de $7,5 \%$, resultaram em filmes mais regulares e íntegros. Devido à facilidade de movimentação das cadeias poliméricas em um meio mais fluido, é possível que estas cadeias tenham se ordenado de uma maneira mais linear e estável, produzindo um filme mais resistente e uniforme.

O parâmetro considerado mais importante para manter a integridade do filme, foi o método de remoção deste da placa formadora. Várias tentativas foram feitas, após secagem overnight do filme em capela química, em diferentes placas formadoras (de metal, plástico e cerâmica), mas todas levavam à ruptura do filme. $\mathrm{O}$ método mais eficiente para remoção de um filme de poliamida íntegro foi através da imersão do filme polimérico recém-formado em um banho de água destilada a temperatura ambiente, promovendo uma inversão de fases. Quando a solução polimérica foi vertida na placa formadora de filme, esta foi imediatamente imergida em um banho de água destilada. Sendo a poliamida um polímero apolar, esta foi imediatamente solidificada em contato com a água (não-solvente), formando assim um filme polimérico íntegro, mais regular e já solto da placa formadora, o qual foi retirado do 
recipiente e acondicionado para secagem. A Figura 2 ilustra o filme polimérico obtido pelo método de inversão de fases.

Figura 2 - Filme de poliamida obtido através da imersão em não-solvente; (a) pouco nãosolvente; (b) imersão completa em não-solvente.

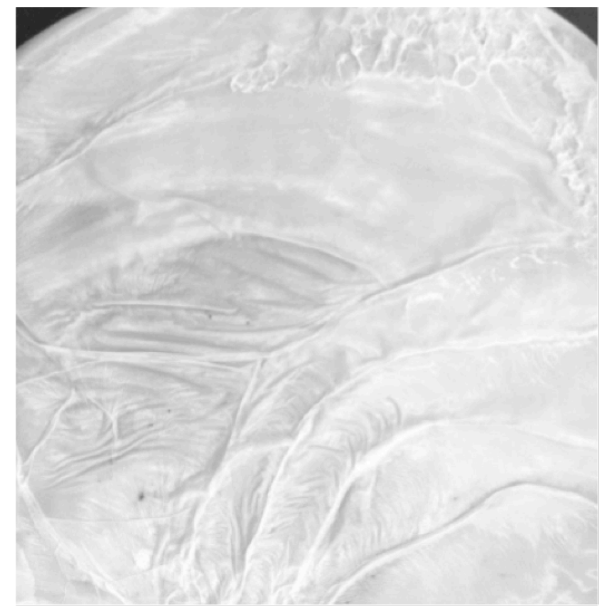

(a)

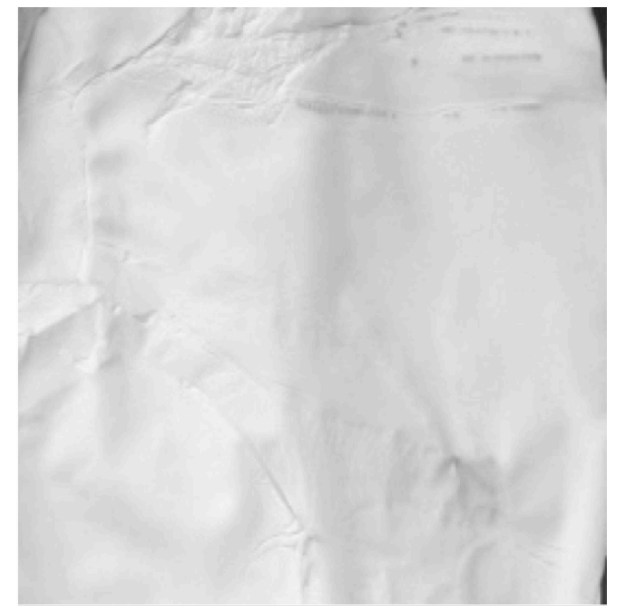

(b)

Com objetivo de melhorar a formação do filme de poliamida, um agente plastificante foi utilizado. Neste trabalho utilizou-se a glicerina $\left(\mathrm{C}_{3} \mathrm{H}_{5}(\mathrm{OH})_{3}\right)$, com a vantagem de ser mais amigável ao meio ambiente, bem como por possuir uma composição similar ao pentaeritritol $\left(\mathrm{C}\left(\mathrm{CH}_{2} \mathrm{OH}\right)_{4}\right)$, que é um composto utilizado no preparo de agentes antichama (Liang t. Al. 2013, Fanglong et. Al. 2013, Horrocks, 2016 b).

A glicerina, em concentração de $10 \%$, foi adicionada à solução de poliamida, antes da formação do filme. Os resultados comparativos foram analisados de forma qualitativa e foi possível perceber a melhora imediata na resistência mecânica do filme. Este apresentou um aspecto mais liso e elástico, melhorando as características de resistência ao rasgo do filme polimérico.

Com o filme de poliamida adequado, este trabalho seguirá com a funcionalização do filme, buscando propriedade de resistência a chamas. Desta forma, segue o estudo em busca de um aditivo composto por fósforo e nitrogênio (Xie et. Al., 2014, Liang et. Al., 2013, Horrocks, 2016 b), como o poli(fosfato de amônio) $\left[\mathrm{NH}_{4} \mathrm{PO}_{3}\right]_{\mathrm{n}}$ e a melamina $\left(\mathrm{C}_{3} \mathrm{H}_{6} \mathrm{~N}_{6}\right)$, a fim de aprimorar as propriedades de resistência ao calor do filme de poliamida.

\section{CONCLUSÃO}

Apesar de a produção de filmes ser um assunto amplamente debatido na literatura, foi um desafio reproduzir em laboratório os métodos já estudados de formação de filme. Com o objetivo de melhorar a resistência de filmes de poliamida, estes foram produzidos utilizando o método de inversão de fases, para transformação de soluções poliméricas em filmes solidificados. Este método se mostrou eficiente, rápido e seguro, por se tratar de um nãosolvente aquoso. Os filmes produzidos por este método apresentaram textura uniforme, área superficial intacta e deformação elástica mais acentuada. Com isso, espera-se utilizar esta 
metodologia para dar continuidade aos estudos de produção de filmes e fibras de poliamida de alto desempenho, através da incorporação de aditivos funcionais, como os aditivos antichama.

\section{REFERÊNCIAS}

CROWN E. M.; BATCHELLER J. C., Technical textiles for personal thermal protection. Handbook of technical textiles, p. 271-281, Editora Woodhead, 2ª ed., 2016.

DEOPURA B. L.; ALAGIRUSAMY R.; JOSHI M.; GUPTA B, Polyesters and Polyamides, p. 609, Editora Woodhead, 2008.

FANGLONG Z.; QUN X. QIANQIAN F.; RANGTONG L. KEJING L., Onfluence of nanosilica on flame resistence behavior of entumescente flame retardant cellulosic textiles: remarkable synergistic effect? Surf. \& Coat. Techn., v. 294, p. 90-94, 2016.

HORROCKS A. R., Technical textiles in transport (land, sea and air). Handbook of technical textiles, p. 325-354, Editora WoodHead, $2^{\mathrm{a}}$ ed., 2016, a.

HORROCKS A. R., Technical fibers for heat and flame protection. Handbook of technical textiles, p. 325-354, Editora WoodHead, $2^{\mathrm{a}}$ ed., 2016, b.

LIANG S.; NEISIUS N. M.; GAAN S., Recent development in flame retardant polymeric coatings. Prog. In Organ. Coat., 76, p. 1642-1665, 2013.

MEDEIROS K. M. de; MORAIS D. D. S.; KOJUCH L. R.; LIRA H. L., Avaliação do comportamento térmico de membranas planas de poliamida/ argila obtidas pela técnica de inversão de fases. Rev. Eletr. De Mat. e Proces. V8.1, p. 36-43, 2013.

RAJENDRAN S.; ANAND S. C.; RIGBY A. J., Textiles for healthcare and medicinal application. Handbook of technical textiles, p. 135-164, 2a ed., 2016.

RAWAL A.; SHAH T. H.; ANAND S. C, Geotextiles in civil engineering. Handbook of technical textiles, p. 111-130, Editora WoodHead, 2a ed., 2016.

XIE C.; ZENG B.; GAO H. YITING X.; WEIANG L. XINYU L.; LIZONG D.. Improving thermal properties of epoxy resins by a novel reactive phosphorous-containing curing agent. Pol. Eng. And Science, V 54, p. 1192-1200, 2014.

YUVARAJ K. S.; KUMAR R., Challenges in designing protective garments. Contemporary issues and trends in fashion, retail and management. Reflections-2014, p 584-594. 2014. 